\title{
Die italienischsprachigen Handschriften der Sächsischen Landesbibliothek - Staats- und Universitätsbibliothek Dresden
}

Neue Perspektiven der Forschung

Herausgegeben von

Anna Katharina Plein und Markus Schürer

unter redaktioneller Mitarbeit von

Wiebke Gerlach und Anika Herber 
Die italienischsprachigen Handschriften der Sächsischen Landesbibliothek Staats- und Universitätsbibliothek Dresden

Herausgeber: Anna Katharina Plein / Markus Schürer

Entstanden im Rahmen des DFG-geförderten Projekts „Erschließung und Digitalisierung der italienischsprachigen Handschriften der SLUB Dresden“

Zitierfähige URL: https://nbn-resolving.org/urn:nbn:de:bsz:14-qucosa2-708522

DOI: https://doi.org/10.25366/2020.09 


\section{Inhalt}

\section{Anna Katharina Plein / Markus Schürer}

Die Sächsische Landesbibliothek - Staats- und Universitätsbibliothek

Dresden (SLUB) und ihre italienischsprachigen Handschriften.

Eine Einführung

Maria Lieber / Christoph Oliver Mayer

Die italienischen Handschriften in Dresden - eine Kontextualisierung in der Hofkultur

Serenella Baggio

Gli italiani a Dresda. Varietà linguistiche nella Collezione sassone

Michele Coscia

Oralità nella predicazione medievale: l'esempio della Leggenda di sant'Antonio abate conservata presso la SLUB (Mscr.Dresd.Ob.6)

Eef Overgaaun

Handschriften von Dantes Divina commedia in Berlin und Dresden

Adriana Paolini

Scritture svelate. Il manoscritto della Commedia di Dresda

Umberto Dassi

Ignote terzine di Dante in un codice delle tragedie di Seneca

(Mscr.Dresd.Dc.152)

Fabio Forner

Petrarca a Dresda: dai manoscritti alle stampe

Marica di Pietro

Il codice Mscr.Dresd.Ob.21: una possibile collocazione in area mantovana

Giuseppe Mollo

La genesi di Delle fortificationi di Carlo Theti con particolare riguardo ai manoscritti dresdensi (Mscr.Dresd.Ob.14, Ob.15 e Ob.16-17) 
Matteo Guidetti

Il testimone sassone della Cortona convertita. Un percorso catalografico

Rashid-S. Pegah

Handschriftliche Libretti von Domenico Lalli oder: von Neapel über

Venedig und Arolsen nach Delhi

Fabio Marri

Un approccio alle raccolte poetiche-drammatiche tra Sei e Settecento

Personenregister

Register der zitierten Handschriften 


\title{
Oralità nella predicazione medievale: l'esempio della Leggenda di sant'Antonio abate conservata presso la SLUB (Mscr.Dresd.Ob.6)
}

\author{
Michele Coscia
}

Obiettivo di questo studio è la presentazione di alcuni risultati delle ricerche da me effettuate ${ }^{1}$ relativamente alla Leggenda di sant'Antonio abate conservata presso la Sächsische Landesbibliothek - Staats- und Universitätsbibliothek (SLUB) di Dresda, nell'intento di mettere in rilievo attraverso un'attenta analisi codicologica e linguistica la possibilità che tale testo fosse usato come supporto alla predicazione in lingua volgare. Esso costituisce la terza parte del codice Mscr.Dresd.Ob.6, ${ }^{2}$ uno degli otto manoscritti quattrocenteschi in lingua italiana conservati presso la SLUB ${ }^{3}$ descrittosecondo le norme della Deutsche Forschungsgemeinschaft (DFG) nell'ambito del progetto "Erschließung und Digitalisierung von Handschriften in italienischer Sprache der SLUB” (2015-2021).

Nella prima parte di questo contributo si presenteranno alcuni dati codicologici relativi al manoscritto analizzato, mentre nella seconda parte ci si concentrerà sull'analisi dell'interpunzione, della grafia, della fonetica, della morfologia e della sintassi, dando soprattutto spazio a quei risultati che possano illuminarci nella ricerca del contesto religioso-culturale, della provenienza geografica e delle possibili modalità d'impiego di questo testo nel Medioevo.

Cito in questo articolo i prelievi dal testo del manoscritto indicandoli con il numero di carta $^{4}$ e di riga.

\section{Parte I: Elementi codicologici ${ }^{5}$}

Il codice, conservato presso la SLUB di Dresda sotto la segnatura Mscr.Dresd.Ob.6, è un manoscritto composito, comprendente tre distinte unità codicologiche: I (ff. 1-56), II (ff. 57-

\footnotetext{
1 La Leggenda di sant'Antonio abate conservata nel Mscr.Dresd.Ob.6 è stata altresì oggetto della mia Tesi di Laurea Magistrale (Relatore: Prof. Dr. Maria Lieber; Correlatore: Prof. Serenella Baggio), elaborata nell'ambito del progetto di Doppia Laurea Dresda-Trento.

2 SLUB Dresden, Mscr.Dresd.Ob.6.

3 Il corpus dei codici in lingua italiana conservati presso la SLUB presenta un solo manoscritto prequattrocentesco, risalente al XIV secolo (Pedron [2013], pp. 27-28).

$4 \quad$ In apice al numero di carta pongo $r$ per recto, $v$ per verso, $a$ per la colonna di sinistra e $b$ per quella di destra.

5 Mi limito in questa sede a presentare solo i più importanti dati codicologici unitamente a quelli che possano dare un contributo alla discussione relativa all'origine e all'uso del testo della Leggenda. Per approfondimenti rimando alla descrizione codicologica effettuata da Burkhard Krieger (Krieger: Descrizione di
} 
119) e III (ff. 120-132). L'unità codicologica meglio conservata e leggibile è la terza; il testo della seconda - al contrario di quello della prima - è, nel complesso, in buono stato. Tutta la prima unità codicologica, infine, presenta danni causati dall'acqua (presumibilmente dovuti agli eventi bellici che hanno interessato Dresda nel 1945), che rendono di difficile lettura molte carte (particolarmente rovinate sono le ff. 11-13 e 27-50). Il codice, di una certa consistenza (I + 132 ff. + II) e grandezza (281 - 287 x $195-206$ mm), è interamente cartaceo e presenta filigrane in tutte e tre le unità codicologiche (undici tipologie di filigrana nella prima unità codicologica; due nella seconda e due nella terza), la cui analisi ${ }^{6}$ suggerisce una datazione attorno agli anni Trenta del Quattrocento. Tale datazione, da prendere cum grano salis (si tratta infatti di mera corrispondenza delle filigrane presenti ai modelli conosciuti e catalogati), apparrebbe confermata dall'analisi della scrittura. Il tipo di scrittura del testo della prima e della seconda unità codicologica, riconducibile probabilmente alla medesima mano, è infatti quello della mercantesca libraria, databile alla prima metà del XV sec.; ${ }^{7}$ il testo della terza unità codicologica (ff. 120-132), vergato da una mano differente rispetto a quello delle due unità precedenti, è scritto in scrittura semigotica (databile tra fine Trecento e inizio Quattrocento) ed è diviso in due colonne per facciata, eccezion fatta per i ff. $131 \mathrm{v}$ e $132 \mathrm{r}$, nelle quali la divisione del testo in colonne non è presente. Per quanto riguarda l'ornamentazione, limito qui il discorso alla sola terza unità codicologica, ${ }^{8}$ oggetto di questo contributo; in essa, pur non essendovi miniature, si può comunque riscontrare una certa cura formale. Vi sono infatti (ai ff. 120r e 124r) due iniziali decorate di colore rosso, che occupano un'altezza di sei righe; esse costituiscono le iniziali rispettivamente del primo e del secondo capitolo; i paragrafi del testo presentano iniziali di colore rosso e blu (l'alternatività del colore non è regolare) e di altezza pari a tre righe. La lettera con cui inizia il terzo capitolo è di colore rosso e - al contrario dell'altezza delle iniziali dei primi due capitoli - è pari soltanto a tre righe.

Dal punto di vista della descrizione interna va inoltre rilevato come ad ogni unità codicologica corrisponda un'opera: nella prima (ff. 1-56) si trova infatti una Vita di san Girolamo, solo in parte (la prima parte, seppur con molte varianti) corrispondente ad un testo toscano ${ }^{9}$ del XIV sec., pubblicato da Zambrini ${ }^{10}$ e da questi attribuito al pisano Domenico Cavalca, frate appartenente all'Ordine dei Predicatori, vissuto tra il 1270 e il $1342 ;^{11}$ nella seconda unità codicologica (ff. 57-119) si trova, dopo un breve proemio in lingua latina tratto dall'Epistola di san Paolo Apostolo ai Galati (Gal. 5,25-6,10), la Disciplina degli spirituali12 di Domenico Cavalca; nella terza unità codicologica (ff. 120-132) vi è infine la Leggenda di sant'Antonio abate.

Mscr.Dresd.Ob.6) secondo i criteri della DFG e a quella da me effettuata (Coscia [2016], pp. 4-18), rifacendomi alle linee guida per la descrizione dei manoscritti presenti in Petrucci (2001), pp. 81-104.

6 Cfr. Krieger: Descrizione di Mscr.Dresd.Ob.6.

7 Da notare le forme rotonde, tipiche di questa scrittura, particolarmente visibili nelle iniziali, soprattutto in $A$ e $G$.

$8 \quad$ Per la descrizione dell'ornamentazione presente nelle prime due unità codicologiche si rimanda a Krieger: Descrizione di Mscr.Dresd.Ob.6 e a Coscia (2016).

9 Per maggiori informazioni relative a questo testo, vd. Dalarun / Leonardi (2003) vol. II, p. 366.

10 Cavalca: Leggenda di Santo Ieronimo.

11 Cfr. Delcorno (1979), p. 577.

12 L'edizione di riferimento per quest'opera è quella di Bottari (Cavalca: Disciplina degli spirituali col trattato delle trenta stoltizie). 
Quest'ultimo testo, che non sembra corrispondere a nessuno di quelli a noi noti ${ }^{13}$ (neppure a quello della vita di Sant'Antonio Abate del Cavalca ${ }^{14}$ ) è diviso in tre capitoli, il primo dei quali dedicato alla vita del Santo (si parla qui della decisione del Santo di dedicarsi alla vita eremitica e di alcuni eventi prodigiosi), il secondo alla translatio del suo corpo dall'Egitto a Costantinopoli e l'ultimo infine dedicato alla seconda translatio (da Costantinopoli a Vienne, in Francia).

Per quanto concerne la storia del codice è importante sottolineare la presenza in tutte e tre le unità codicologiche di note di possesso, databili alla seconda metà del XVI sec., recanti il nome Oratio di Lionardo Busini, personaggio legato alla diplomazia veneziana; questi infatti presso la famiglia Contarini di Venezia "aveva iniziato la sua carriera come prelato e avvocato" e, dopo i viaggi nelle due capitali europee, "risiedette in Roma come un membro della Congregazione Urbana" ${ }^{15}$ Come rileva anche Krieger, ${ }^{16}$ notizie più certe su questo codice si possono avere soltanto a partire dal XVIII sec.; è, infatti, tra il 1721 e il 1733 che il conte Christian Heinrich von Watzdorf (1698-1747), ciambellano alla corte sassone di Augusto il Forte, entrò in possesso del codice, portandolo con sé in Sassonia. Dopo la morte di Watzdorf (1747) la sua biblioteca venne acquistata dal conte Heinrich von Brühl, il quale ricoprì tra il 1746 e il 1751 la carica di primo ministro del re Augusto III. Nel 1768 la Kurfürstliche Bibliothek in Dresden acquistò per 50000 talleri la biblioteca di Brühl, assegnando negli anni successivi a questo manoscritto l'attuale segnatura Ob.6.

\section{Parte II: Analisi linguistica}

Il fatto che il testo analizzato sia stato concepito come supporto alla predicazione in lingua volgare e, quindi, sia vicino alle ragioni dell'oralità e della retorica omiletica, ci spingono a concentrare l'attenzione sull'analisi linguistica e della punteggiatura, che - unitamente all'uso delle maiuscole - può recare preziose tracce di indicazioni per la lettura. Tutto ciò trova altresì una conferma esterna - per analogia - nel fatto che nel primo Trecento viene messo in atto in Italia (in particolar modo in Toscana) "quel programma di diffusione della cultura religiosa, che gli Ordini mendicanti perseguono con la predicazione volgare e con la traduzione dei più importanti monumenti della letteratura sacra", che trova nell'opera del domenicano Cavalca - autore della Disciplina degli spirituali trascritta nella seconda unità codicologica di questo manoscritto - "il momento più felice". ${ }^{17}$

\section{La punteggiatura}

Vista la relativa abbondanza di segni paragrafematici nel testo, l'analisi della punteggiatura è stata effettuata, censendo tutti i segni, riportandoli in tabelle, nelle quali si menziona altresì il numero di carta e di riga, il contesto (vengono trascritte almeno la parola che precede e quella

\footnotetext{
13 Cfr. Dalarun / Leonardi (2003), Vol. II, p. 61-64. Per le somiglianze con altri testi relativi alla vita di sant'Antonio si veda Krieger: Descrizione di Mscr.Dresd.Ob.6.

14 Cfr. Cavalca: Vite dei Santi Padri.

15 Portoghesi (2008), p. 10.

16 Krieger: Descrizione di Mscr.Dresd.Ob.6.

17 Delcorno (1979), p. 579.
} 
che segue il segno analizzato) ed altre indicazioni utili (se esso si trova in discorso diretto o indiretto oppure a fine riga) a comprendere la ratio che abbia guidato tale usus distinguendi.

L'analisi ha dato innanzi tutto modo di rilevare la seguente gerarchia dei segni $<$. $>$ (42 occorrenze), $</>(34),<\cdot>(8),<\cdot / />(6),<. />(1),<,>(1)$.

Non soffermandomi in questa sede nell'esposizione dettagliata dell'analisi di ogni segno di interpunzione, ${ }^{18}$ ma cercando di guardare all'insieme dei segni interpuntivi presenti nella terza unità codicologica si può notare come essi si pongano ancora in una logica intermedia tra quella che vede come loro scopo il supporto alla lettura (con l'indicazione delle pause vocali) e quella che mira ad evidenziare e distinguere le strutture logico-sintattiche; quest'ultimo ruolo della punteggiatura, che si affermerà a partire dagli ultimi anni del Quattrocento con le scelte editoriali adottate da Aldo Manuzio, trova in Petrarca ed in Boccaccio i suoi precursori. A quest'ultimo in particolare va - come evidenzia Coluccia nel suo saggio sulla punteggiatura nei volgari d'Italia in riferimento al codice Hamiltoniano del Decameron - "il merito di essersi spinto più decisamente nella direzione della modernità", ${ }^{19}$ operando una ponderata razionalizzazione del sistema interpuntivo. Egli, infatti, ha ridotto l'uso del punto nel corpo del periodo e, soprattutto, all'interno della proposizione; ha altresì ridotto l'uso altrimenti massiccio della virgola (sperimentandone anche la variante grafica moderna: <,>), rendendola un segno adatto a sfumare partizioni logiche e sintattiche minori, ed ha sperimentato nuovi segni, come l'accento del vocativo <ó $>$, che si affermeranno solo successivamente grazie anche alla diffusione della stampa. ${ }^{20}$ Si prospetta, dunque, interessante capire, sulla base dell'analisi qui condotta, in quale direzione si muova il testo della Leggenda di sant'Antonio abate presente nel codice Mscr.Dresd.Ob. $6^{21}$ in relazione alla punteggiatura. Sulla base dell'analisi da me compiuta sui singoli segni, si può affermare che, pur muovendoci in un contesto in cui la punteggiatura è ancora considerata un supporto alla lettura more antiquo, ${ }^{22}$ vi sono comunque alcuni elementi che introducono una concezione più moderna di punteggiatura, volta in particolare a distinguere le partizioni logico-sintattiche del discorso. Gli elementi di novità, presenti nel testo analizzato, più importanti sono: 1) cinque casi in cui il punto assume funzioni moderne ${ }^{23}$ (in due casi segna la fine del periodo, in un caso la fine di capitolo, una sola volta la fine di paragrafo e una volta la fine di una rubrica; in ben quattro di questi cinque casi segue la lettera maiuscola); 2) l'utilizzo di una virgola di forma grafica moderna $<,>$; 3) la presenza (in un solo caso) di un segno (il punto seguito dalla virgola: $<. />$ ) per introdurre il discorso diretto. Si può, dunque, notare come da un lato molti elementi rimandino ad una visione della punteggiatura legata alle esigenze della lettura ad alta

\footnotetext{
18 Per tale analisi dettagliata si veda Coscia (2016), pp. 19-26 e 154-157.

19 Coluccia (2008), p. 90.

20 Coluccia (2008), pp. 93-94.

21 E'opportuno ricordare qui che il codice, sulla base delle filigrane presenti nelle carte e di altri elementi, è databile alla prima metà del Quattrocento.

22 Si vedano, ad esempio, l'uso del punto per indicare una pausa nella lettura all'interno di una proposizione e il largo uso della virgola per racchiudere alcuni monosillabi.

23 Normalmente nel testo analizzato la fine del periodo è altrimenti segnalata non già dalla presenza del punto (come oggi comunemente si usa), ma da una spaziatura più ampia (maggiore o uguale a $4 \mathrm{~mm}$; altrimenti lo spazio tra le parole, se presente, è di 2-3 mm), a cui spesso segue la lettera maiuscola.
} 
voce $^{24}$ e come dall'altro vi siano alcuni casi che, seppur nella loro rarità, costituiscono uno slancio verso quel modus distinguendi che, trovando per alcuni aspetti i suoi precursori illustri in Petrarca ed in Boccaccio, si affermerà con successo solo a partire dal Cinquecento per impulso della stampa.

Vi è infine da rilevare la presenza in due punti del testo (in f. 127vb, 14 ed in f. 128va, 38) di un segno di richiamo $\left\langle^{\wedge}>\right.$ collocato nell'interlinea inferiore in corrispondenza di un'aggiunta nell'interlinea superiore. Ciò testimonia la particolare attenzione del copista alla segnalazione di ritocchi nel testo trascritto; simili accorgimenti editoriali si trovano anche in alcuni codici autografi del Petrarca. ${ }^{25}$

\section{Grafia}

Nel compiere l'analisi della grafia si è cercato soprattutto di evidenziare - attraverso lo studio della grafia del testo - quali scelte grafiche fossero improntate a criteri etimologici (in voga soprattutto a partire dagli inizi del Quattrocento) e quali a criteri di tipo fonetico, ${ }^{26}$ nella consapevolezza che tali criteri "non procedono separatamente, ma [...] si intrecciano di frequente, con l'effetto di una continua oscillazione all'interno di uno stesso testo". ${ }^{27}$

Tale analisi ha messo in particolare evidenza due usi grafici interessanti ai fini della nostra analisi sull'uso e la provenienza del testo della Leggenda: il primo legato all'utilizzo delle maiuscole ed il secondo riguardante i fenomeni di apocope. In primis l'uso, seppur sporadico, delle maiuscole distintive, cioè di una parola o di un gruppo di parole scritte con lettere tutte maiuscole allo scopo di "rendere distinti determinati dispositivi testuali". ${ }^{28} \mathrm{Nel}$ testo analizzato, infatti, se ne trovano quattro casi: la parola, con cui inizia il primo capitolo (STANDO f. 120ra, 3); ${ }^{29}$ il gruppo di parole posto alla fine del primo capitolo (DEO GRA(TLA)S $A M E N$ - f. 124ra, 3) e la prima (NEL - f. 124ra, 8) ${ }^{30}$ e l'ultima parola del secondo capitolo (AMEN - f. 131ra, 7). La presenza della maiuscola distintiva, seppur nella sua rarità, costituisce un elemento di elaborazione stilistica, atto a segnalare (unitamente all'uso dell'inchiostro rosso nel caso della parola $A M E N$ ) l'inizio o la fine del testo di un determinato capitolo. La natura di analoghi inserti distintivi, con una funzione paratestuale, negli autografi del Boccaccio è oggetto di studio da parte di Marco Cursi, il quale segnala la presenza di 92 esempi

\footnotetext{
24 Tali esigenze si possono ancor meglio comprendere, se si pensa al fatto che molto spesso le vite dei Santi (in particolar modo quelle dei Patres Ecclesiae) erano usate come supporto alla predicazione (cfr. Delcorno [1979], p. 579).

25 Vd. Coluccia (2008), p. 86.

26 Precisa giustamente Nicoletta Maraschio nella prima nota del suo saggio sull'evoluzione e codificazione della grafia che "quando si dice fonetico a proposito di un sistema grafico si deve intendere fonologico, perché in realtà normalmente sono solo gli elementi distintivi ad essere 'trascritti”" (Maraschio [1993], p. 140).

$27 \quad$ Maraschio (1993), p. 159

28 Cavallo (1996), p. 23

29 In questo caso la prima lettera della parola $(S)$ è un'iniziale decorata; le lettere che seguono sono, invece, delle maiuscole non decorate.

30 La lettera $N$ è un'iniziale decorata; le lettere EL sono, invece, maiuscole non decorate.
} 
di maiuscola distintiva, distribuiti in sei codici autografi del Boccaccio su diciotto. ${ }^{31}$ Quello presente nel testo della Leggenda di sant'Antonio abate è un utilizzo della maiuscola distintiva che, nella sua rarità (solo quattro casi, tutti nel primo e nel secondo capitolo; il terzo capitolo non presenta questo fenomeno), da un lato ricorda quello presente in alcuni testi di Petrarca, ${ }^{32}$ il quale "utilizza con grande parsimonia brevi sequenze di maiuscole in funzione distintiva in posizione incipitaria, nella parola posta ad inizio d'opera, per le lettere che seguono l'iniziale miniata" ${ }^{33}$ (nel testo da noi analizzato si vedano le parole STANDO e NEL con cui si aprono rispettivamente il primo ed il secondo capitolo), dall'altro (per i casi presenti in f. 124ra, 3 e in f. 131 ra, 7) ${ }^{34}$ si hanno analogie con l'uso del Boccaccio, autore che utilizza la maiuscola distintiva soprattutto nelle espressioni di introduzione o conclusione di opera o di sezioni di essa. ${ }^{35}$

In secondo luogo, per quanto concerne i fenomeni di apocope, risulta essere di particolare interesse (soprattutto ai fini di una più precisa collocazione geografica del testo della Leggenda nel territorio toscano) il caso di apocope sillabica in $<\mathrm{u}>$ (ŬBI - ff. 120va, 32, 123rb, 2 e 125vb, 4); la forma $<\mathrm{u}>$ da ŬBI latino costituisce un tratto tipico del toscano occidentale $\mathrm{e}$, in particolare, del pisano. ${ }^{36} \mathrm{E}$ ' altresì forma del toscano occidentale l'apocope di $\langle\mathrm{e}\rangle$ nel monosillabo bivocalico $<$ due $>^{37}$ presente in f. 120va, 25 ed in f. $127 \mathrm{ra}, 3$ in cui si trova la forma $<\mathrm{du}>$, la quale resta nel testo analizzato comunque minoritaria. ${ }^{38}$

Si registra, infine, l'apocope sillabica d'origine aplologica nel sostantivo citta (CIVITĀTEM; venti occorrenze, p. es. in f. 120vb, 29 e in una sola occorrenza la forma non apocopata cittade (nell'espressione incittade; f. 129ra, 30); a fianco a questi casi, il testo presenta cinque casi con la grafia cipta (f. $121 \mathrm{ra}, 21$ ), falsa rietimologizzazione, la quale si trova in alcuni testi lucchesi antichi. ${ }^{39}$ Va infine segnalato il fatto che non si registrano casi del tipo cita(de) con la scempia - che invece sono tipici del dialetto di Pisa. ${ }^{40}$

31 Cursi precisa che "quasi il $90 \%$ delle occorrenze sono raccolti in due soli testimoni: lo Zibaldone Laurenziano 29.8 che ne vanta 21 (vale a dire il $26 \%$ del totale) e la Miscellanea Laurenziana 33.31, nella quale se ne contano addirittura 51 (cioè il $62 \%$ del totale)" (Cursi [2013], p. 64).

32 Si veda, ad esempio, Vaticano Barb. lat. 3358, f. 4r (cfr. Cursi [2013], p. 64).

33 Cursi (2013), p. 64 (la citazione è tratta dalla nota a piè di pagina numero 107).

34 Si tratta delle parole DEO GRA(TIA)S AMEN ed $A M E N$ con cui vengono conclusi rispettivamente il primo ed il secondo capitolo della Leggenda di sant'Antonio abate.

35 Una differenza tra l'esempio boccaccesco e il testo presente nella terza unità codicologica del codice Mscr.Dresd.Ob.6 risiede nel fatto che in quest'ultimo non compaiono mai espressioni quali incipit, explicit, finit o simili, che invece caratterizzano tutti i casi di utilizzo da parte del Boccaccio di maiuscola distintiva in posizione d'inizio o fine opera o di sezioni di essa (cfr Cursi (2013), pp. 66-68).

36 Vd. Castellani (1980), p. 291 e 311.

37 Vd. Castellani (1980), p. 311.

38 Si riscontrano infatti 23 casi con $<$ due $>$ (ad esempio in $121 \mathrm{ra}, 9)$ a fronte dei due che presentano la forma tipica dei dialetti toscano-occidentali $<\mathrm{du}>$.

39 Vd. Castellani (1980), p. 324.

$40 \quad$ Nel saggio Pisano e lucchese, nel quale si cercano di evidenziare le "differenze che separano l'antico pisano dall'antico lucchese" (Castellani [1980], p. 283) coll'intento di “assegnare con sicurezza all'uno o all'altro dialetto i testi che finora si definivano "pisano-lucchesi»" (Castellani [1980], p. 283), l'autore menziona il caso del sostantivo città: esso, infatti, "nella maggior parte dei testi pisani [è scritto] costantemente con la $t$ scempia" (Castellani [1980], p. 323); nel dialetto di Lucca, invece, "si ha citta”" (Castellani [1980], p. 324) o varianti - attestate in testi lucchesi antichi - come cictà e ciptà (Castellani [1980], p. 324). Cfr. anche Castellani (1980), p. 306. 


\section{Fonetica}

Il testo della Leggenda presenta, anche per quanto concerne gli usi fonetici, un'oscillazione tra forme fiorentine ed esiti riconducibili all'area dialettale toscano-occidentale (Lucca e Pisa). Per quanto concerne il vocalismo tonico vi sono infatti sia elementi tipicamente fiorentini, come la prevalenza delle forme dittongate anche in huomo/uomo (8 casi con dittongo e 4 senza) e dopo consonante seguita da $<\mathrm{r}>$ (brieve, priegho/priego), ${ }^{41}$ che tratti appartenenti al tipo occidentale, come la conservazione di $<\mathrm{au}>$ davanti a $<\mathrm{l}>$ in Paulo e taule ("tavole"). ${ }^{42}$ L'anafonesi, fenomeno proprio anticamente "solo di fiorentino, pratese, pistoiese, lucchese, pisano e volterrano (e non dei dialetti centrali e orientali: senese, aretino e cortonese)", ${ }^{43}$ è presente con regolarità nel testo nei suoi due diversi tipi:

1. /e/ del latino volgare (in cui erano confluiti $\overline{\mathrm{E}}$ ed $\breve{\mathrm{I}}$ del latino classico) $>$ /i/, nei casi in cui /e/ tonica è davanti a / KK/ (-LJ-) e a /nj/ (-NJ-); il fenomeno appare in tutti i casi in cui è previsto, come, per esempio, in $\mathrm{fa}$ miglia (FAMILLIAM) f. 131v, 37 ed in piglia (*PİLIARE 123ra, 3);

2. /e/ del latino volgare (in cui erano confluiti $\overline{\mathrm{E}}$ ed $\breve{\mathrm{I}}$ del latino classico) $>$ /i/, /o/ del latino volgare $(\breve{U} / \bar{O})>/ \mathrm{u} /$ nei casi in cui /e/ ed /o/ toniche si trovano davanti ad una nasale velare, ossia davanti ai nessi NG e NC del latino; il fenomeno non presenta irregolarità. Si veda ad esempio lingue (LINGUAE) 122 $2^{\text {va }}, 12$ e lungha (LŎNGAM) f. 127rb, 28.

Per quanto concerne il vocalismo atono basti qui citare due interessanti risultati. Riguardo alla labializzazione della vocale protonica davanti a consonante labiale, anche nei casi in cui il fiorentino antico conosce la labializzazione, il testo analizzato tende a mantenere le forme con la vocale palatale: per il verbo dimandare - con la /i/ - si registrano nel testo ventuno occorrenze (p. es. in f. 121 ra, 38; f. 124vb, 39 ed in f. 126vb, 36); compare, inoltre, l'aggettivo simigliante (f. 131v, 22). Mentre per il primo lemma si trovano nella Leggenda due casi in cui la labializzazione è avvenuta (domandolli - f. 124va, 3 e domandi-f. 126ra, 35), per il secondo non vi sono forme labializzate opponibili. Si ricordi che, al contrario che nel fiorentino, nel pisano e nel lucchese antichi "non si ha [...] il passaggio di $i(e)$ a $o$ in dimandare, dimane, [...] simigliare". ${ }^{4}$ Tipica della Toscana occidentale èinoltre "la presenza di /u/ da /o/ protonica o intertonica in varie voci nelle quali il fiorentino mantiene $/ \mathrm{o} /$ "; 45 nel testo analizzato si registrano quattro attestazioni di questo fenomeno in f. 120va, 32, in f. 123rb, 2 ed in f. 125vb, 4, dove si ha la forma la forma $u(\mathrm{UBI}),{ }^{46}$ ed in f. $131 \mathrm{ra}, 21$, dove è presente la locuzione $u v^{2}{ }^{47}$ (AUT VĒRUM). Alla forma $u$ - sia quella derivante da ǓBI che quella derivante da AUT - attestata in testi pisani antichi, ${ }^{48}$ si possono opporre forme con il regolare

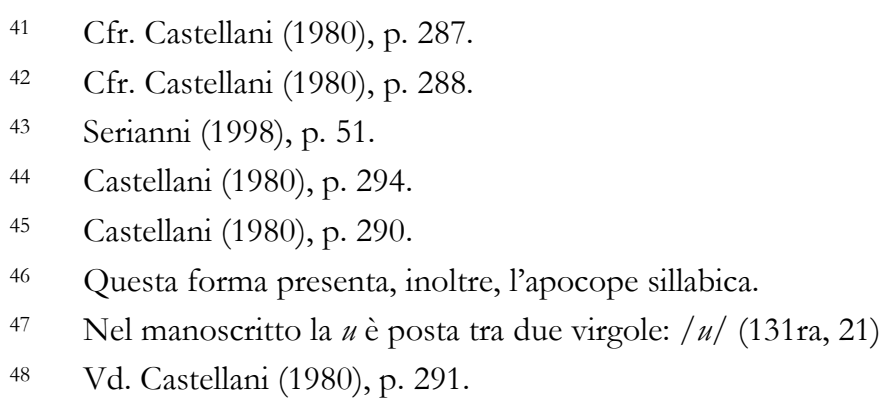


/o/ fiorentino: ove (f. 125ra, 36) overo (ff. 120vb, 10; 123va, 4; 131 rb, 35) e o vero (f. $131 \mathrm{rb}$, 23).

Anche l'analisi del consonantismo rivela una compresenza di esiti fiorentini e toscanooccidentali: si ha, ad esempio, la sonorizzazione della velare sorda iniziale in parole per le quali il fiorentino non conosce questo fenomeno; queste forme, tipiche della Toscana occidentale, ${ }^{49}$ sono le seguenti: gamello (f. $121 \mathrm{va}, 8$ ), ghamello (f. $121 \mathrm{vb}, 17$ ) e ghamelli (f. $121 \mathrm{rb}, 25$ e altri quattro casi). A questi casi il testo oppone solo una forma con la velare sorda: chamelli (f. 121 va, 38). Inoltre la perdita (per influsso settentrionale) dell'elemento occlusivo nelle affricate [t乃] e [dz], che costituisce "il tratto più importante dei due dialetti [pisano e lucchese ]", ${ }^{50}$ si riscontra nel testo analizzato in alcune forme, come ad esempio meso ${ }^{51}$ (f. $122 \mathrm{vb}$, 25) forse ( "forze"; f. 123vb, 6 e 125ra, 20), vechiessa" (f. 123vb, 39 - f. 124ra, 1), palasso ${ }^{53}$ (ff. 124rb, 17 e 130va, 13), certessa $a^{54}$ (f. 126rb, 24), pussa (f. 127vb, 34), soppi (f. 128rb, 39), i(n)nansi (una sola occorrenza: f. 129rb, 28) e pesso (f. 130rb, 36). Non si trovano nel testo forme da opporre (che mantengano l'elemento occlusivo) agli ultimi due casi. Agli elementi tipici dell'area toscano-occidentale vanno aggiunti alcuni esiti particolari del verbo LAXARE; il Castellani indica infatti tra $\mathrm{i}$ tratti tipici dei dialetti toscani occidentali il passaggio " $/ \mathrm{ks} />$ /ss / in lassare (anche - in Toscana - a Volterra, San Gimignano, Siena, Cortona, accanto a lasciare e minoritariamente ad Arezzo-Sansepolcro) e, almeno a Pisa, in masella" ${ }^{55}$ Nel testo della Leggenda le forme con /ss/ prevalgono nettamente: si ha, infatti, lassali ('lasciali' f. $121 \mathrm{rb}, 32$ ), lassero ('lascierò' - f. 123ra, 22), lassi ('lasci' - f. 123rb, 28), lassiate ('lasciate' f. 123vb, 17) e lassase ('lasciasse' - f. 124ra, 14); si oppone a queste esclusivamente la forma lasciato (f. 123va, 6).

\section{Morfologia}

L'analisi della morfologia dei nomi (comuni e propri), degli aggettivi, degli articoli, delle preposizioni, dei pronomi, dei verbi e degli avverbi conferma la presenza nel testo - anche a livello morfologico - di forme fiorentine e di forme toscano-occidentali. Esulando dagli obiettivi di questo contributo la trattazione sistematica di tale analisi, riporto a titolo di esempio solamente due casi. In primis per quanto riguarda la prima persona singolare del verbo avere, va notato come il testo proponga, accanto alla forma $o$ ('ho', p. es. f. $121 \mathrm{ra}$, 3), anche quella toscano-occidentale $a b b o^{56}$ (una sola occorrenza - f. 120rb, 34). Il secondo esempio riguarda la situazione di alcuni avverbi nel testo della Leggenda: va infatti notata la netta prevalenza della forma tipica dei dialetti toscani occidentali - presente anche nel dialetto antico di Pistoia ${ }^{57}$ - quine (diciassette occorrenze) su qui, che trova solo quattro attestazioni nella

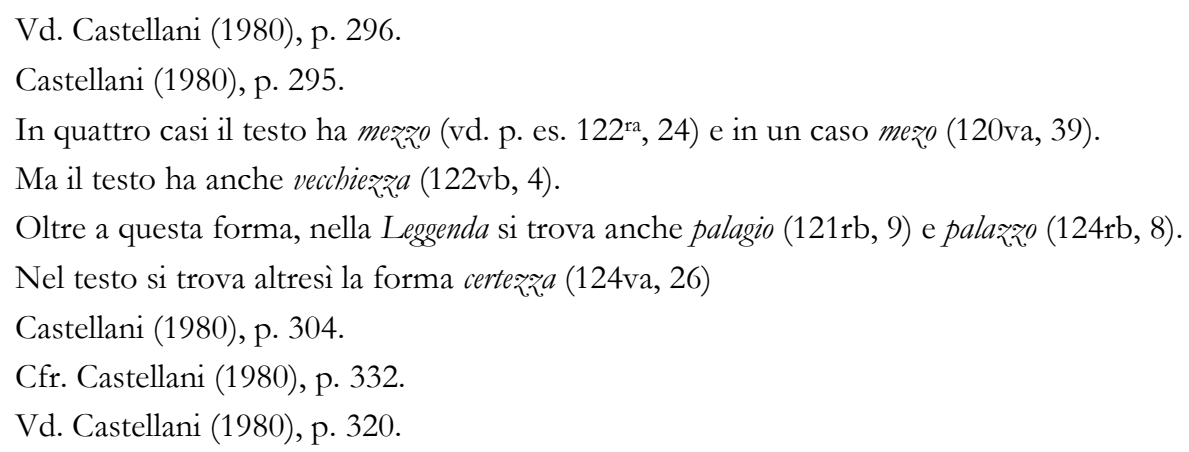


Leggenda, e il doppione degli avverbi di tempo infine (dodici occorrenze) ed infino (due occorrenze), $\mathrm{i}$ quali si alternano anche in qualche testo pisano antico. ${ }^{58}$ Notevoli sono, inoltre, le forme anco $^{59}$ (presente solo in f. 131rb, 18) e fuora (un'unica occorrenza: f. 129vb, 23). A quest'ultima forma, tipica del dialetto pisano antico, ${ }^{60}$ possiamo opporre fuori, forma la quale, ben più presente nel testo (otto occorrenze; p. es. f. $121 \mathrm{rb}, 31$ ), è tipica del fiorentino. ${ }^{61} \mathrm{Da}$ notare, infine, l'occorrenza dell'avverbio forsi ('forse'; presente solo in f. 122va, 9-10), forma presente nel pisano (accanto a quella forse, ${ }^{62}$ la quale non è però attestata nella Leggenda), nel lucchese e nel pistoiese antichi ${ }^{63}$ e dell'avverbio temporale avale ('ora'), tipico dei dialetti toscani occidentali. ${ }^{64}$

\section{Sintassi}

La sintassi presenta chiare tracce del fatto che questo testo, elaborato in ambiente religioso (quindi in uno degli ambiti della società medievale maggiormente acculturati), era destinato alla predicazione. Il testo, infatti, se da un lato non rinunzia ad una certa cura formale (non si tratta, infatti, di una sorta di raccolta tematica di appunti per la predicazione, ma di un testo agiografico di supporto a quest'ultima), dall'altro risponde alle esigenze dell'oralità e del discorso in pubblico, ${ }^{65}$ coniugate con quelle della retorica omiletica: la prosa della Leggenda è caratterizzata da una prevalenza di una sintassi paratattica ${ }^{66}$ che ben si adatta alle esigenze orali della predicazione; numerose sono quindi le congiunzioni copulative, in particolare et, e sono relativamente numerosi i casi in cui quest'ultima si trova a inizio frase con l'iniziale maiuscola, talvolta dopo una spaziatura doppia. ${ }^{67}$

Il modello di tale sintassi paratattica, propria di molti testi agiografici e adatta al racconto in pubblico di episodi della vita dei santi, può essere riscontrato in certi passi della Bibbia e, in particolare, del Nuovo Testamento; spiega infatti Maurizio Dardano nella prima parte del recente testo da lui curato sulla sintassi dell'italiano antico, parlando degli aspetti sintattici degli scritti agiografici in volgare, che bisogna "considerare l'influsso della Bibbia, che si avverte nella diffusione di una sintassi paratattica presente negli exempla come nella predicazione

\footnotetext{
58 Cfr. Castellani (1980), p. 320.

59 Spiega la Manni che la forma anco per anche è "comune alla maggior parte della Toscana" e che "si trova solo sporadicamente in testi fiorentini prequattrocenteschi di carattere non letterario", sostenendo che la sua presenza nel fiorentino rinascimentale, oltre che all'influenza dei dialetti circostanti, "può essere dovuta all'influsso della forma manco di cui, proprio nel secolo XV, si diffonde l'uso avverbiale” (Manni [1979], p. 165).

60 Manni (1979), p. 168.

61 Va precisato che anche a Firenze si andò diffondendo nel Quattrocento e, soprattutto, nel Cinquecento la forma fuora in luogo di quella più antica fuori, probabilmente per influenza del dialetto pisano, nel quale tale forma era presenta già in epoca molto antica (cfr. Manni [1979], p. 168).

62 Il testo presenta due occorrenze dell'omografo forse ("forze"), per il quale vd. supra 2.3.3-b).

63 Castellani (1980), p. 318.

64 Castellani (1980), p. 204 e p. 217.

65 Per il rapporto tra predicazione (e dunque oralità) e punteggiatura, vd. supra 2.1.

66 È bene qui precisare che considerare la paratassi "sempre e comunque [...] come un segno di imperizia è un pregiudizio superato da tempo" (Dardano [2012], p. 29).

67 In totale il testo offre centouno casi di Et con l'iniziale maiuscola, in ventitré dei quali questa lettera è posta dopo una spaziatura doppia.
} 
(dove si allea alla ripresa del parlato)". ${ }^{68}$ Questi testi - prosegue Dardano - agiografici dunque "badano soprattutto al senso" ${ }^{69}$ e sono altresì caratterizzati da "strutture periodali adatte alla presentazione-celebrazione di santi" formule di apertura e di chiusura [che] hanno un carattere stereotipico e sono spesso il tramite per la diffusione di latinismi". ${ }^{71}$ Tutto questo sembra esser vero anche per la Leggenda di sant'Antonio abate, nella quale si notano, oltre che una struttura sintattica generalmente paratattica, numerose preghiere in discorso diretto, aperte da formule caratterizzate dal vocativo cui segue immediatamente l'imperativo che, in modo positivo o negativo (si ha in questo caso il non $^{72}$ tra l'allocutivo alla divinità invocata e il verbo), esprime la richiesta (p. es. signore idio no(n) dare allebe | stie lanime che i(n) te siconfidano - f. 120vb, 3-4 - e Signore idio levati | eaintaci p(er)lotuo s(an)c(t)o nome - f. 120vb, 6-7); le formule finali, con cui si concludono i tre capitoli recano sempre espressioni latineggianti o parole in lingua latina tipiche della tradizione liturgica. ${ }^{73} \mathrm{La}$ sintassi è quella tipica dei testi in prosa dell'italiano antico, del quale rispetta le norme riguardanti la struttura delle frasi. Queste ultime infatti presentano le caratteristiche della prosa dell'ultima parte del Trecento e, in generale, dell'italiano preumanistico.

In tutte le proposizioni interrogative dirette presenti nel testo della Leggenda si attua la c.d. inversione del soggetto, come per esempio in f. $129 \mathrm{va}, 7-8$ : $p$ (er)che cia|vete voi celato cosi pretioso tesauro[?].

Si trovano, inoltre, nella Leggenda esempi di anticipazione dell'oggetto diretto, un fenomeno facoltativo ma frequente nell'italiano antico; in questi casi il testo analizzato - in accordo con la norma sintattica di allora - riprende con un pronome clitico l'oggetto anteposto, qualora quest'ultimo sia in posizione di Topic o Cornice $^{74}$ (e dunque vi sia del materiale tra l'oggetto diretto e il verbo flesso): $i$ vostri facti dal | parlare liguardate (f. 125rb, 31-32).

Qualora invece l'oggetto diretto anteposto si trovi immediatamente prima del verbo flesso, esso ha le proprietà di un operatore e dunque, in questi casi, si registra l'assenza di

\footnotetext{
68 Dardano (2012), p. 26.

$69 \quad$ Dardano (2012), p. 16.

$70 \quad$ Dardano (2012), p. 25.

$71 \quad$ Dardano (2012), p. 25.
}

72 Paola Benincà, parlando della periferia sinistra della frase nell'italiano antico, spiega che la negazione può "funzionare come un clitico, e aggiungersi così alla testa verbale e agli altri eventuali clitici, oppure [...] occupare la posizione di sintagma (come un pronome debole [...])" (Benincà / Poletto [2010], p. 29), precisando che, qualora la negazione funzioni come un clitico (primo caso), essa sarà in proclisi (mai in enclisi), e che, se invece occupa la posizione di sintagma, può trovarsi "anche in posizione iniziale assoluta di frase" (Benincà / Poletto [2010], p. 29), potendo altresì fungere "da appoggio ai clitici che la seguono" (Benincà / Poletto [2010], p. 29 ), non essendo (in questo secondo caso) essa stessa un clitico.

73 A conclusione del primo capitolo si ha la formula latina in maiuscola distintiva DEO GRA(TLA)S AMEN (124ra, 3); il secondo, invece, si conclude con le parole Ad laude et glo | ria del nostro singnore (Yesu) (Cristo) al $\mid$ quale e lonore et lagloria insecu|la seculo(rum). A M E N (131 ra, 4-7); le ultime parole del terzo capitolo sono invece le seguenti: Concedendocelo lo | nostro singnore (Yesu) (Cristo) che colpadre ecolfigliuolo et collo sp(irit)o santo | vive et rengna didio intrinita $p\left(\right.$ er)fecta $p$ (er) tucti isechuli deseculi se |culo(rum) Amen (132r ${ }^{\mathrm{r}}$, 16-19). Da notare in quest'ultimo caso il pleonastico seculo(rum), che sembra esser di troppo rispetto alla verisimilmente ispiratrice formula latina, che suona per omnia saecula saeculorum.

74 Quindi nelle posizioni $T_{1}$ o $T_{3}$. 
copia pronominale (al contrario di ciò che si verifica nell'italiano moderno, che in questi casi prevede comunque la ripresa con il clitico ${ }^{75}$ );

p. es. Ma s(er)penti drago|ni et $\mathrm{i}(\mathrm{n})$ numerabili leoni tigri et pardi |Topi come volpi aspidi basilischi et | unicorni trovono infiniti (f. 127ra, 5-8).

Il testo, inoltre, offre esempi di "un fenomeno essenzialmente sintattico", ${ }^{76}$ quello del "sì demarcatore", ${ }^{77}$ che corrisponde al "pro-sintagma con proprietà di costituente" $" 78$ di cui parla Benincà. Questo monosillabo, derivante da SİC latino, compare nei seguenti punti della Leggenda:

a) f. 122vb, 22-23: Conpiuta ladima(n)da sidisse beato paulo hora e del mangiare (si noti qui come il sì connetta quanto espresso nella frase implicita alla sua sinistra con quanto si trova alla sua destra; il soggetto si trova regolarmente subito dopo il verbo);

b) f. 123va, 27-30: Et | beato antonio siritorno inella cella | sua co(n)grande bumilita p(er)severa $(n)$ do $\mid i(n) p($ er)fecta oratione infine che visse (in questo caso il soggetto, posto immediatamente prima di sì, viene tematizzato da quest'ultimo ${ }^{79}$;

c) f. 128ra, 19-21: Et sille dicea quel dan(i)el adalta | voce Sanctissimo co(n)fessore no(n)mi tor $\mid m(e n)$ tare (qui il sì, occupando la posizione di operatore altrimenti vuota, impedisce l'enclisi; ${ }^{80}$

d) f. 124vb, 39-125 $5^{\text {ra }}$, 3: Iosare senpre co(n) || loro et leturbationi del mare no(n) teme $|\mathrm{ra}(n) n o|$ Et sille parole langiolo a(n)nuntio | alveschovo teophilo (notiamo come qui il si tematizzi quanto espresso dalla frase precedente $\left.{ }^{81}\right)$.

\footnotetext{
75 Per la trattazione di questa differenza tra l'italiano antico e quello moderno, vd. Benincà / Poletto (2010), p. 34.

76 Baggio (2014), p. 41. In questo articolo l'autrice, dopo aver fornito e analizzato vari esempi di questo tipo di sì, soffermandosi sulla natura anaforica e cataforica del monosillabo, ne sottolinea la continuità d'uso fino all'età contemporanea, in particolare in testi di natura non letteraria dell'Italia settentrionale.

77 Baggio (2014), p. 38. Suggerisce Serenella Baggio nel suo articolo che sia meglio evitare di parlare di questo come si pleonastico, in quanto la sua presenza, "non priva di significato e funzione" (Baggio [2014]), p. 37), è sì facoltativa, ma non pleonastica.

78 Benincà / Poletto (2010), p. 50.

79 Per il soggetto tematizzato, vd. Baggio (2014), p. 40 e Benincà / Poletto (2010), p. 51. Si ricordi che "sì può essere preceduto da altri costituenti, che hanno una funzione pragmatica di tema, o di cornice situazionale della frase, o di collegamento col testo precedente" (Benincà / Poletto [2010], p. 50).

80 Riguardo al ruolo sintattico di sì per evitare l'enclisi cfr. Egerland / Cardinaletti (2010), p. 433; Baggio (2014), p. 42 e Benincà / Poletto (2010), p. 52.

81 A questo proposito osserva la Benincà che il "sì anteposto nella periferia ha l'effetto di far risultare come tema l'elemento che lo precede a sinistra; quando non c'è niente che preceda sì, si deve intendere che il tema è lo stesso della frase precedente" (Benincà / Poletto (2010), p. 52).
} 
Nel testo della Leggenda si trovano, inoltre, due attestazioni di or senza il valore deittico di "adesso"; non avendo "valore semantico pregnante", ${ }^{82}$ questo or svolge una funzione sintattica analoga a quella del si demarcatore, ${ }^{83}$ collocandosi nella posizione immediatamente precedente al verbo, ${ }^{84}$ il quale a sua volta si trova nella posizione di testa a sinistra del soggetto. Le uniche due frasi del testo analizzato (entrambe interrogative dirette) ${ }^{85}$ in cui questo monosillabo compare sono:

a) f. 120vb, 27-30: Ornonavea dei mo|nim(en)ti i(n) quella nostra terra oinella | citta nostra checcia co(n)ducti admorire | i(n) questa solitudine;

b) f. 128vb, 5-6: orse tu vivo lume degli | ochi nostri.

Or può altresì precedere l'elemento interrogativo o cooccorrere con sì ma di questi due casi il nostro testo non presenta alcun esempio.

\section{Conclusioni}

Come mostrano alcuni elementi (in modo particolare l'analisi della scrittura e delle filigrane) dell'analisi codicologica, riportati nella prima parte di questo articolo, l'unità codicologica contente la Leggenda di sant'Antonio abate risulta essere databile al XV sec., forse agli anni Trenta del Quattrocento. L' analisi della punteggiatura presente nel testo, che è stato da me trascritto cercando di essere "sensibili all'importanza di conservare la punteggiatura originale, con tutte le sue anomalie e le 'incongruenze' (piuttosto apparenti che sostanziali, se si ricorre alla categoria dell'oralità)" ${ }^{86}$ ha messo in evidenza come da un lato alcuni segni interpuntivi abbiano la funzione antica di fornire indicazioni per la lettura e dall'altro lato vi siano tuttavia degli elementi d'innovazione (l'uso - seppur sporadico - del punto nella funzione di concludere il periodo; la presenza di una virgola di foggia moderna e la presenza, in un caso, di un segno interpuntivo per indicare l'inizio del discorso diretto), che si affermeranno solo nel Cinquecento grazie alla stampa, evidenziando inoltre alcune analogie nell'uso dei paragrafematici tra il testo della Leggenda e i manoscritti autografi di Petrarca e di Boccaccio. Guardando poi alla grafia e alla morfologia del testo, si può riscontrare una compresenza di forme tipicamente fiorentine e di tratti appartenenti ai dialetti toscano-occidentali. La storia del codice non ci fornisce elementi per pensare ad una sua origine lucchese o pisana; questa compresenza di forme fiorentine e forme toscano-occidentali, pur ricordando la generale influenza del tipo occidentale sul dialetto di Firenze soprattutto dopo la peste di metà Trecento, potrebbe lasciare spazio all'ipotesi di un'origine toscano-occidentale del testo. Tale ipotesi, che, stando ai dati di cui siamo finora in possesso, deve restare tale, appare comunque molto suggestiva, se si pensa che nella Pisa del Trecento si trovava un convento domenicano (quello

\footnotetext{
82 Benincà / Poletto (2010), pp. 52-53.

83 Cfr. Baggio (2014), p. 38.

84 Questo tipo di or può anche non precedere immediatamente il verbo, fungendo in questo caso "da sostituto di un elemento con funzione di Cornice” (Benincà / Poletto [2010], p. 53).

85 A proposito del caso in cui or introduce le frasi interrogative dirette, nota la Benincà come tale funzione ricordi quella "di o introduttivo delle interrogative del fior. mod." (Benincà / Poletto [2010], p. 53).

86 Baggio (2000), p. 22.
} 
di Santa Caterina) che fin dalla seconda metà del Duecento "godeva di una notevole fama", 87 tra le cui mura nel 1272, per suggerimento di Tommaso d'Aquino, vi veniva stabilito un corso di Artes. Questo convento, dove si formò e visse anche il frate dell'Ordine dei Predicatori Domenico Cavalca, ospitava inoltre una delle più antiche biblioteche domenicane, ricca di opere sia religiose che classiche, e un ben organizzato scriptorium; questi elementi concorsero a fare di questo luogo "un centro adatto all'insegnamento e alla predicazione" 88 e ad "iniziative di volgarizzazione della cultura" ${ }^{89}$ analoghe a quelle che hanno prodotto il testo della Leggenda.

$87 \quad$ Delcorno (1979), p. 577.

88 Delcorno (1979), p. 577.

$89 \quad$ Delcorno (1979), p. 577. 


\section{Bibliografia}

Biblioteca Apostolica Vaticana (Città del Vaticano) ms. Vaticano Barb. lat. 3358

Biblioteca Medicea Laurenziana (Firenze) ms. Zibaldone Laurenziano 29.8

Biblioteca Medicea Laurenziana (Firenze) ms. Miscellanea Laurenziana 33.31

SLUB Dresden, Mscr.Dresd.Ob.6

Staatsbibliothek zu Berlin - Preußischer Kulturbesitz, Ms. Ham. 90

Cavalca, Domenico: Disciplina degli spirituali col trattato delle trenta stoltizie, ed. Giovanni Bottari, Roma (Stamperia di Niccolò e Marco Pagliarini) 1838 [1757]

Cavalca, Domenico: Vite dei Santi Padri, ed. Carlo Delcorno 2 voll., Firenze (Edizioni del Galluzzo) 2009

Cavalca, Domenico: Leggenda di Santo Ieronimo, ed. Francesco Zambrini, Imola (Tipografia Galeati) 1852

Baggio, Serenella: La sintassi orale nella punteggiatura: tre casi, in: Rivista italiana di dialettologia, 24 (2000), pp. 7-28

Baggio, Serenella: Se pareba nell'Indovinello veronese: altri casi di SİC iniziale, in: Quaderni di filologia romanza della facoltà di Lettere e Filosofia dell'Università di Bologna, 22 (2014), pp. 37-55

Benincà, Paola / Poletta, Cecilia: L'ordine delle parole e la struttura della frase, in: Giampaolo Salvi / Lorenzo Renzi (a cura di): Grammatica dell'italiano antico, 2 voll., Bologna 2010, vol. I, pp. 27-75

Castellani, Arrigo: Pisano e lucchese, in: Arrigo Castellani: Saggi di linguistica e filologia italiana e romanza, tomo I, Roma 1980, pp. 283-326

Castellani, Arrigo: Grammatica storica della lingua italiana. vol. I. Introduzione, Bologna 2000

Cavallo, Guglielmo: Iniziali, scritture distintive, fregi. Morfologie e funzioni, in: Cesare Scalon (a cura di): Libri e documenti d'Italia: dai Longobardi alla rinascita delle città. Atti del Convegno nazionale dell'Associazione Italiana Paleografi e Diplomatisti (Cividale del Friuli, 5 - 7 ottobre 1994), Udine 1996, pp. 15-33

Coluccia, Rosario: Teorie e pratiche interpuntive nei volgari d'Italia dalle origini alla metà del Quattrocento, in: Bice Mortara Garavelli (a cura di): Storia della punteggiatura in Europa, Roma / Bari 2008, pp. 65-98

Coscia, Michele: Mscr.Dresd.Ob.6: la leggenda di Sant'Antonio abate conservata presso la Sächsische Landesbibliothek - Staats- und Universitätsbibliothek (SLUB) di Dresda, Tesi di doppia laurea inedita, Dipartimento di Lettere e Filosofia, Università degli Studi di Trento a.a. 2015-2016 Cursi, Marco: La scrittura e i libri di Giovanni Boccaccio, Roma 2013

Dalarun, Jaques / Leonardi, Lino et al. (a cura di): Biblioteca agiografica italiana. Repertorio di testi e manoscritti, secoli XIII-XV, Firenze 2003 
Dardano, Maurizio (a cura di): Sintassi dell'italiano antico. La prosa del Duecento e del Trecento, Roma 2012

Delcorno, Carlo: "Cavalca, Domenico", in: Dižionario biografico degli italiani, Vol. 22, Roma 1979, pp. 577-586

Egerland, Verner / Cardinaletti, Anna: I pronomi personali e riflessivi, in: Giampaolo

Salvi / Lorenzo Renzi (a cura di): Grammatica dell'italiano antico, 2 voll., Bologna 2010, vol. I, 401-467

Krieger, Burkhard: Descrizione di Mscr.Dresd.Ob.6, in: Manuscripta Mediaevalia, URL: http://www.manuscripta-mediaevalia.de/dokumente/html/obj31594809 (25.11.2019)

Manni, Paola: Ricerche sui tratti fonetici e morfologici del fiorentino quattrocentesco, in: Studi di grammatica italiana, 8 (1979), pp. 115-171

Maraschio, Nicoletta: Grafia e ortografia: evoluzione e codificazione, in: Luca Serianni / Pietro Trifone (a cura di): Storia della lingua italiana, vol. I, I luoghi della codificarione, Torino 1993, vol. I, pp. 139-227

Pedron, Chiara Maria: Mscr.Dresd.Ob.8: Dialogo consolatorio di Giannozzo Manetti. Avvio di uno studio sul patrimonio manoscritto italiano a Dresda, Tesi di doppia laurea inedita, Dipartimento di Lettere e Filosofia, Università degli Studi di Trento a.a. 2012-2013

Petrucci, Armando: La descrizione del manoscritto. Storia, problemi, modelli, Roma 2001

Portoghesi, Paolo et al.: Borromini e gli Spada. Un palazzo e la committenza di una grande famiglia, Roma 2008

Serianni, Luca: Lezioni di grammatica storica italiana, Roma 1998 [1988] 
